\title{
Niños y niñas transgéneros: ¿nacidos en el cuerpo equivocado o en una sociedad equivocada?
}

Transgender children: born in the wrong body or in the wrong society?

Ximena de Toro*

\begin{abstract}
Resumen
Algunos niños/as tienen una identidad de género que difiere de su sexo biológico. Ellos y ellas, los niños/as transgéneros, son a menudo vistos como problemáticos dado que amenazan la construcción de género binaria predominante en la sociedad. Sin embargo, en vez de ser una amenaza, los niños/as transgéneros son parte de la diversidad humana y nos hace comprender la identidad de género como un conjunto de posibilidades. El objetivo de este ensayo fue explorar en el desarrollo de los niños transgéneros a la luz de influyentes teorías, enfatizando sus limitaciones y fortalezas, junto con reflexionar sobre algunos elementos a considerar en el espacio terapéutico con niños transgéneros en el contexto escolar. Para ello se examinó qué es lo que se conoce actualmente acerca de los niños/as transgéneros y las dificultades que ellos y ellas tienen que enfrentar en las distintas etapas de su desarrollo.
\end{abstract}

Palabras claves: niños transgéneros, identidad de género, diversidad humana, terapia, escuela

\begin{abstract}
Some children have a gender identity that does not match their anatomical sex. Trangender children are often seen as problematic because they threaten the binary construction of gender embedded in the society. However, rather than a threat, transgender children are part of the human diversity, and make us understand that gender identity is a fluid continuum. The aim of this essay was to explore in the transgender child's development in light of relevant theories, emphasizing their limitations and assertiveness, in addition to some elements to consider when counselling transgender children in a school setting. This essay examined what is currently known about transgender children and the difficulties that they have to cope with in the different stages of their development.
\end{abstract}

Key words: transgender children, gender identity, human diversity, counselling, school

* Trabajadora Social de la Pontificia Universidad Católica de Chile; Magíster en Estudios de Infancia de la Universidad de Edimburgo; Diplomada en Niñez y Políticas Públicas; Diplomada en Intervención en Abuso Sexual Infantil; Diplomada en Género, familia y políticas públicas. Actualmente es parte del equipo nacional de Chile Crece Contigo del Ministerio de Desarrollo Social. Ha ejercido como docente y ha colaborado en investigaciones vinculadas a familia e infancia. 
Una vida es todo lo que tenemos y vivimos según como creemos que hay que vivirla. Pero, sacrificar lo que eres y vivir sin creer, es un destino más terrible que la muerte.

Juana de Arco (n.d)

\section{Introducción}

El propósito de este ensayo es examinar el desarrollo psicosocial de los niños y niñas transgéneros, brindando ciertas orientaciones a los terapeutas que trabajan con ellos en el contexto educacional. El argumento basal es que las teorías propias de la psicología del desarrollo, tales como la teoría de Erik Erikson, son insuficientes y limitantes para comprender y trabajar con la diversidad de niños, niñas y adolescentes. Por lo tanto, otras corrientes teóricas, junto con un rol más amplio del terapeuta involucrado en el sistema de salud o en el contexto educacional se requiere para trabajar con ellos y ellas. Los especialistas concuerdan en que muy poco es conocido acerca de cómo ayudar a los niños transgéneros (Brill y Pepper, 2008; Kennedy y Hellen, 2010; Wester, McDonough, Maureen, Vogel, y Taylor, 2010), aunque existen mayores probabilidades que ellos se vean envueltos en conductas que amenacen su vida (Grossman y D'Augelli, 2007).

Este ensayo es altamente relevante en la discusión generada hoy sobre cómo abordar la equidad de género en el contexto educacional, tema que cobra importancia a partir del auge de movimientos sociales apuntando a un mayor respeto de la diversidad sexual y de géneros, y la inclusión de dichos elementos en la agenda pública. Desde la experiencia profesional de quien escribe este artículo, llama la atención como la realidad de los niños transgéneros ha surgido como una nueva temática dentro del SENAME e instituciones educacionales, sin tener necesariamente los profesionales de dichas instituciones las herramientas para abordar esta realidad, teniendo como foco el respeto intrínseco a los derechos de niños y niñas. Cabe destacar cómo los medios de comunicación han levantado distintas historias de niños transgéneros y sobre cómo sus padres y colegios han reaccionado, debiendo los primeros lidiar con la discriminación del sistema escolar. Junto a ello un conjunto de películas han aportado a la discusión y a la comprensión de la realidad de los niños, niñas y adolescentes transgéneros ${ }^{1}$, levantando la reflexión sobre la temática.

Sin embargo, la inexistencia de un cuerpo de investigación relevante sobre la temática en español o que no se conozcan muchos niños transgéneros en Chile, no significa que no existan, sino que da cuenta de cómo el tema se invisibiliza, esconde, desconoce y niega. Nos encontramos con padres que no saben cómo enfrentar las demandas de sus hijos de desarrollarse acorde a su propia identidad de género y no de acuerdo a la identidad asignada al nacer, con el riesgo de caer en dinámicas de malos tratos; colegios que obligan a los niños a vestirse de una manera distinta a cómo los niños se sienten; y

\footnotetext{
${ }^{1}$ Algunas películas son: Mi vida en rosa (Francia, 1997); Los chicos no lloran (Estados Unidos, 1999); Tomboy (Francia, 2011)
} 
niños y niñas que desconocen qué les está pasando por no contar necesariamente con el apoyo de adultos con conocimiento de que su proceso es normal y responde a la diversidad humana. En definitiva, la existencia de niños y niñas que viven una infancia de negación, malos tratos y rechazo simplemente por comportarse como ellos y ellas se sienten es, sin duda, un tema que debe ser considerado por la agenda pública.

Primero, este ensayo proveerá antecedentes generales del tema, ilustrando el análisis con un caso real vinculado a la experiencia profesional de la autora, caso que, de acuerdo a la revisión bibliográfica, no difiere de las experiencias que los niños transgéneros enfrentan en otros contextos (Brill y Pepper, 2008; Mallon y DeCrescenzo, 2006; Malpas, 2011). Asimismo, este caso permite reflexionar sobre las consecuencias de no garantizar dentro del sistema público un trato y una atención psicológica pertinente y oportuna a todos los niños y niñas transgéneros, independiente de sus ingresos. Esta atención debiese ser un derecho frente a las dificultades que han de enfrentar en comparación a otros niños que se sienten cómodos con el género asignado al nacer.

Posteriormente este ensayo explorará los aspectos significativos del desarrollo de los niños transgéneros, junto con las barreras que deben enfrentar en el proceso de formación de su identidad. Este análisis se hará mirando críticamente la teoría de Erick Erikson. Siguiendo este análisis crítico, se presentarán algunos elementos del enfoque centrado en la persona, cuyo principal referente es el psicólogo Carl Rogers, destacando la pertinencia de dicho enfoque para el trabajo con niños transgéneros en un contexto terapéutico, específicamente en el medio escolar. Finalmente, en la última parte de este ensayo se darán a conocer algunas reflexiones.

\section{Género, infancia y diversidad}

En diversas sociedades, y por muchos años, se ha concebido como normal una clasificación binaria de las identidades de género, comprendida como una extensión de los genitales con los que una persona nace. Asimismo, y siguiendo a Brill y Pepper (2008), para muchas personas los términos "género" y "sexo" son lo mismo. Esta idea se ha vuelto tan común en las sociedades occidentales y raramente es cuestionada. Sin embargo, sexo y género son diferentes, y el género no necesariamente está conectado con nuestra anatomía. El sexo es algo biológico e incluye aspectos relativos a nuestras hormonas, cromosomas, aparato reproductivo y los genitales, aspectos que son utilizados al nacimiento para identificar a una persona como hombre o mujer. Para estos autores, el género es más complicado y refiere a la compleja interrelación entre los rasgos físicos y nuestro sentido interno como hombre, mujer, ambos o ninguno, así como a las conductas asociadas a ese sentido interno. Nuestra identidad de género puede entonces ser la misma o diferente de nuestro sexo.

El género es entendido como un principio básico de organización de la vida social y para la asignación de deberes, derechos y poderes (Acker, 2004) a partir de su vinculación con significados, representaciones sociales y funciones asociadas a las diferencias físicas, las cuales influyen directamente en las maneras de comportarnos y en nuestras subjetividades. Su importancia radica en que se nos enseña sobre el género desde el 
momento de nacer. Expectativas y mensajes sobre cómo ser y comportarse de acuerdo al género nos bombardean constantemente. Nuestra crianza, la cultura, los pares, la comunidad y los medios de comunicación son algunas de las múltiples influencias que forman nuestra comprensión de qué es el género, aspecto central de nuestra identidad. Siguiendo a Brill y Pepper (2008) las interacciones entre los padres y sus hijos/as que hacen alusión al género comienzan tan pronto cuando el sexo del bebé es conocido. Finalmente, cómo aprendemos e interactuamos con el género desde la primera infancia influye directamente en la forma de ver el mundo de hoy.

Sin embargo, y contrario a algo natural, el género es un concepto construido socialmente. Lo que entendemos por ser hombre o mujer varía a través de las culturas y periodos históricos, como así también varían los atributos considerados "femeninos" o "masculinos". Al igual que otras construcciones sociales, el género es monitoreado de cerca por la sociedad. Prácticamente a todo en la sociedad se le asigna un género. Los juguetes, los colores, la ropa y los comportamientos son algunos de los ejemplos más evidentes. Sin embargo, es importante comprender que la típica vinculación entre el rosado y las niñas, así como entre el azul y los niños, son nociones relativamente nuevas en nuestra historia (Brill y Pepper, 2008).

Distintos movimientos a lo largo del mundo, como el movimiento queer, han cuestionado esta visión binaria, luchando por una concepción más amplia del género, y han dado la batalla por una progresiva integración de las personas que no se ajustan a los estereotipos de género y a las expectativas que la sociedad tiene sobre los hombres y mujeres (Martínez-Guzmán y Montenegro, 2010; Waites, 2010). Estos movimientos han sido claves en levantar la discusión y normalizar el que algunaspersonas tengan una identidad de género que no coincide con su sexo anatómico.

Si bien las personas transgéneros han logrado salir a la luz pública, la existencia de niños y niñas transgéneros, esto es, niñas que sienten, piensan, hablan y se comportan como niños, y niños que sienten, piensan hablan y comportan como niñas, sigue siendo un tema tabú. Para muchos, puede ser incluso inconcebible, como si el ser transgénero fuera una transformación decidida en la vida adulta, aunque la literatura señala que las personas transgéneros se dan cuenta que su identidad de género no coincide con su sexo biológico generalmente en la primera infancia (Brill y Pepper, 2008; Kennedy y Hellen, 2010; Mallon y DeCrescenzo, 2006).

Niños y niñas transgéneros son a menudo vistos como problemáticos (Kennedy y Hellen, 2010), dado que amenazan una construcción de género tan enraizada en nuestras sociedades que asimila una identidad de género con una genitalidad indicada, así como también amenazan una visión de la infancia entendida como una etapa de la vida desvinculada de la sexualidad. En definitiva, son vistos como problemáticos porque cuestionan una imagen que entiende a los niños como seres moldeables por los adultos, no cómo sujetos que pueden cuestionar también los parámetros sociales.

Esto sugiere que existe una invisibilización y negación de la diversidad de género en la primera infancia. Sin embargo, contrario a una amenaza, los niños transgéneros son parte de la diversidad humana, y evidencian cómo la identidad de género, más que una 
construcción estática y binaria, es un abanico de posibilidades (Malpas, 2011).

\section{¿Atrapados en el cuerpo incorrecto?: Niños transgéneros y la adversidad del contexto.}

María tenía 12 años cuando fue derivada a un centro de diagnóstico debido a serios problemas de conducta que gatillaron un episodio de violencia bidireccional entre ella y su madre. La primera impresión al ver a María era que se estaba frente a un niño. Nadie podía dudarlo. Al preguntarle su nombre, María se refería a sí misma como Mario ${ }^{2}$. De acuerdo a los antecedentes recopilados, desde que tenía tres años de edad, Mario había empezado a mostrar "non-conforming gender mannerisms", o comportamientos y modos de ser que daban cuenta de una disconformidad con el género asignado al nacer y/o comportamientos y modos de ser que no se ajustaban a lo que la sociedad espera para el género femenino. Desde que tenía tres años, él había empezado a sentirse y comportarse como Mario. Contrario a las creencias existentes, Mario, al tratar de expresarse conforme a como él se sentía, no estaba desafiando la autoridad de sus padres, ni tampoco requería mayor disciplina para comportarse como una niña o estaba pasando por una etapa. Él quería ser un niño y se sentía así.

La prevalencia de niños que presenta diversidad de género, incluyendo los niños transgéneros, se estima es 1 en 500 niños (Brill y Pepper, 2008). Se entiende por diversidad de género aquellas conductas o intereses que trascienden el límite de lo considerado como normal para un determinado sexo biológico asignado a una persona (Brill y Pepper, 2008). No todos los niños que despliegan una diversidad de género llegan a ser adultos transgéneros (Rosin, 2008), o desarrollan una sensibilidad, concientización y un sentir con respecto a su género que es incongruente con su sexo biológico (Wester et al., 2010).

La transgeneridad es una realidad que cruza cultura, razas, religiones y periodos históricos. Contrario a lo que se piensa, esto no es causado por una crisis familiar, por un abuso sexual, por un estilo parental de tipo estricto o liberal. La gente tiende a pensar que es una fase (Rosin, 2008), una elección o incluso una enfermedad. Sin embargo, para algunos niños que expresan una diversidad de género constante en el tiempo, no es una fase ni una elección racional, sino una expresión de la diversidad humana, y si bien no hay claridad con respecto a sus causas, es posible que la transgeneridad pueda ser explicada por razones biológicas más que por componentes sociales o por la historia de vida de una persona (Brill y Pepper, 2008). Sin embargo, a pesar de varios progresos en cuanto a equidad de género, la hostilidad de la sociedad que culpa a los niños y sus padres por no adaptarse a las tradicionales normas de género continúa (Mallon y DeCrescenzo, 2006).

Junto con la distinción entre sexo y género, cabe añadir una segunda distinción entre género y orientación sexual. Esa última se encuentra "determinada por el deseo natural, tanto sexual, como amoroso y erótico; hacia otras personas" (Movilh, 2010, p.7). Esta distinción cobra relevancia dado que el ser transgénero no tiene ninguna relación con la

\footnotetext{
${ }^{2}$ Para hacer referencia al caso expuesto se hará uso del nombre Mario, acorde a la identidad de género del niño. Cabe añadir que los nombres fueron modificados para proteger su identidad.
} 
orientación sexual: "en otras palabras, el hecho de ser y sentirse hombre o mujer no se vincula con el gusto por personas de igual o distinto sexo" (Movilh, 2010, p.10).Así entonces, nos podemos encontrar hombres transgéneros (personas que nacieron con genitales del sexo femenino y que tienen una identidad de género masculina) y que tienen una orientación homosexual (siente atracción hacia los hombres) y otros que pueden tener una orientación heterosexual (siente atracción hacia las mujeres).

Como la homosexualidad en el pasado, ser transgénero continúa teniendo tintes de patologización. Hasta el año 2013, ser transgénero se vinculaba a la categoría de los trastornos sexuales y de la identidad sexual de acuerdo al Manual Estadístico de los Trastornos Mentales de la Asociación Americana de Psiquiatría (DSM-IV). Esta categorización es bastante controversial, pues si bien algunos especialistas distinguen las variaciones en el género como una enfermedad mental, para otros, los síntomas que presentan los niños transgéneros, tales como baja autoestima, automutilación, pobre imagen de sí mismo, abuso de drogas e intentos suicidas (Chen-Hayes, 2001; Grossman \& D'Augelli, 2007) son el resultado de las restricciones que viven, el rechazo de terceros y las estigmatizaciones por parte de sus familias y la sociedad (Conroy, 2010) en vez de características propias de la naturaleza de las personas transgéneros (Mallon \& DeCrescenzo, 2006). La desventaja de dicha clasificación reside además en la negación de la existencia de personas transgéneros sanas y funcionales (Lev, 2005), así como en el negativo impacto que tiene en las formas de comprender la diversidad de género y por ende en las políticas y programas que apuntan a una mayor equidad de género.

Tales controversias son profundizadas por las autoras Martínez-Guzmán y Montenegro (2010) para quienes el debate entre el modelo psiquiátrico y las teorías queers (teorías que han surgido para visibilizar el carácter de construcción social de las identidades de género concebidas como naturales), ha dado pie a un complejo mapa de tensiones, conflictos y acuerdos entre las distintas posiciones, discusión que se encuentra vinculada a un conjunto de "intereses políticos, estrategias de supervivencia y adaptación social, negociaciones y reconfiguraciones" (p.28). De esta manera, para ambas autoras, el transtorno de la identidad sexual, visto desde los lentes de los transconocimientos o de una aproximación alternativa a la cuestión transgénero que se escinde del modelo patológico y que enfatiza las múltiples y complejas posiciones sobre las identidades trans, es:

despojado de su aura esencialista-estigmatizante, para quedar abierto a múltiples cuestionamientos y transfiguraciones pero incorporando las perspectivas, los intereses y las vidas de quienes se relacionan con él en carne propia (Martínez-Guzmán y Montenegro, 2010, p.35).

En la última versión de dicho Manual (DSM-V) se desestima la aparición del llamado trastorno de identidad sexual que asimilaba el ser transgénero con un desorden mental, y pasa a ser una categoría en sí misma, bajo el nombre de disforia de género, para referirse a un descontento cognitivo y afectivo con el género asignado al nacer, siendo definido más específicamente cuando es usado como una categoría diagnóstica (APA, 2013). Lo anterior, pues disforia de género se refiere al estado de angustia o estrés que viene acompañado con la incongruencia del género que la persona manifiesta y el 
género asignado al nacer. Aunque no todos los individuos expresan tal nivel de estrés como resultado de dicha incongruencia, de acuerdo al DSM-V muchos llegan a estarlo si la posibilidad de una intervención física ya sea por hormonas o por cirugía no está disponible (APA, 2013). Según el manual, este término es más descriptivo que el término previo usado en el DSM-IV, pues enfoca el problema clínico en la disforia y no en la identidad (APA, 2013). Sin embargo no deja de ser un término y clasificación controversial. Cobra entonces sentido la postura inclusiva de Martínez-Guzmán y Montenegro (2010, p. 34):

no son las identidades las que deben circunscribirse a los lineamientos teóricos o a los paradigmas de turno, sino la producción de conocimiento la que debe mutar para generar espacios materiales y simbólicos más habitables; es la ciencia la que puede aprender de las rupturas y las excursiones extra-normativas y extraacadémicas que llevan a cabo identidades y prácticas no normativas.

\section{Niños y niñas transgéneros a la luz de las teorías del desarrollo}

El desarrollo es el proceso por el cual un organismo, humano o animal, crece y cambia a lo largo del transcurso de la vida (Smith, Cowie, y Blades, 2003), varía entre un niño y otro, así como también varía el sentido del sí mismo, lo que en inglés se denomina el sense of self. El sentido del sí mismo ha sido ampliamente analizado por diferentes escuelas de pensamiento en la medida que los individuos traen tal sentido del sí mismo a terapia (Brinich y Shelley, 2002).

Para efectos de este ensayo, y con el fin de comprender el desarrollo de los niños y niñas transgéneros, dos conceptualizaciones del sentido del sí mismo serán consideradas y puestas a discusión. La primera, una perspectiva psicoanalítica a la mano de Erik Erikson quien en su teoría propone un sentido del sí mismo maleable (Brinich y Shelley, 2002), enfatizando el proceso de formación de la identidad y el puente entre lo intrapsíquico y lo intersubjetivo (Bohleber, 2010). Segundo, una perspectiva humanista sostenida por Carl Rogers quien sostiene una perspectiva relacional del ser (McMillan, 2004; Hawkins, 2008).

\section{- La infancia: la concientización de la diferencia.}

Mario tenía tres años cuando empezó a sentir una fuerte identificación con niños, prefiriendo estereotipos masculinos de juguetes y actividades. Como todos los niños, Mario trataba de encontrarse y definirse a sí mismo por medio de la identificación con objetos y clamando ciertas características como propias (Bohleber, 2010). Su madre inicialmente lo animaba y trataba de que adquiriera costumbres femeninas, pero sus intentos fueron infructuosos. Al mismo tiempo, ella era criticada por no forzar a su hijo a usar ropa y peinado característicos de las niñas. La familia extensa se sumó a las críticas, rehusando invitar al niño a las fiestas familiares hasta que aceptara usar ropas femeninas. La madre de Mario, confundida sobre cómo criar a su hijo, intentó buscar apoyo psicológico. Fue así como se encontró con un conjunto de profesionales que la llenaron de diferentes consejos pero que no fueron capaces de explicarle qué realmente 
le pasaba a su hijo.

Diversas teorías vinculadas a la psicología del desarrollo han surgido para comprender a los niños y niñas, teniendo dichas teorías una fuerte influencia en nuestras concepciones sobre la infancia, siendo varias de ellas criticadas por tratar a la infancia como una etapa de preparación para la adultez, aminorando el valor que la infancia tiene por sí misma. No obstante lo anterior, la influencia de dichas teorías sigue siendo preponderante, de allí la pertinencia de mirarlas críticamente a la luz de las experiencias de los niños transgéneros.

Una de estas teorías más influyentes ha sido la teoría psicosocial de las ocho etapas de desarrollo humano de Erik Erikson ${ }^{3}$. Cada una de estas etapas o estadios psicosociales se caracteriza por contar con una crisis o un conflicto que deben enfrentar los individuos.

Con respecto a la primera etapa, que abarca desde el nacimiento hasta los dos años, Erikson sugiere que si el afecto y los cuidados no son suficientes, el niño crece desconfiando de sí mismo y de las otras personas. Esta etapa depende principalmente del cuidado y el afecto entregado por los cuidadores (Hopper, 2007).

La siguiente etapa, desde los dos a los tres años de edad, trata sobre el desarrollo de la autonomía o por el contrario, de la vergüenza y dudas acerca del sí mismo (Hopper, 2007). Analizando esta etapa a partir de la literatura sobre los niños transgéneros, es posible añadir que en este periodo los niños empiezan a desarrollar las habilidades del lenguaje e identificarse con un género, centrando su atención en aquellos estereotipos y modelos del mismo género que los guían cómo actuar (Brill y Pepper, 2008). De acuerdo a Brill y Pepper, la mayoría de las personas tiene una comprensión de su identidad de género entre los dos y tres años de edad, pero incluso antes los bebés pueden distinguir a las personas por su presentación y voz, y rápidamente aprenden de sus pares y los adultos acerca del género de los colores, juguetes, ropas.

Una vez que los niños van adquiriendo conciencia de su identidad de género, van buscando activamente actuar de acuerdo a los modelos por género. A partir de una combinación de los condicionamientos sociales y las preferencias personales, a los tres años la mayoría de los niños prefieren actividades y exhiben comportamientos típicamente asociados con su género. Para la mayoría, esta conciencia de su género permanece estable en el tiempo, y tiende a refinarse aún más con el inicio de la pubertad.

Lo mismo sucede entonces con los niños transgéneros, quienes luchan activamente por actuar y socializar acorde al género con el que se identifican, tal como Mario, quien, según informa su madre, empezó a demostrar sus preferencias vinculadas al género en cuanto empezó a comunicarse. Según lo reportado, Mario tenía las mismas pataletas que Erikson sugiere que los niños despliegan cuando no se les permite seguir adelante con sus planes (Hopper, 2007). Estos sentimientos de frustración son probablemente más experimentados por los niños transgéneros en la medida que los padres o

${ }^{3}$ Para efectos del presente ensayo, el análisis se centrará en las etapas relativas a la infancia. 
cuidadores tienden a desaprobar y rechazar preferencias incompatibles a su sexo biológico, profundizándose de esta forma fuertes conflictos con el niño que no actúa acorde a los estereotipos, generando angustia en los cuidadores y posibles problemas en la pareja. Sin embargo, y siguiendo a Erikson, si los deseos de un niño o niña son continuamente restringidos y los niños tienen que renunciar constantemente a sus deseos para complacer a los padres, es posible suponer que un sentimiento de vergüenza puede surgir de dichas discrepancias.

La siguiente etapa, de los tres a los cinco años, es aquella en donde de acuerdo a Erikson el niño o niña desarrolla la iniciativa o la culpa. Según Erikson (1963, citado en Franz y White, 1985, p.29), en esta etapa las diferencias de género tienden a polarizarse a partir de una socialización de los roles asignados para cada sexo biológico: "boys and girls are differentiated not only by differences in organs, capacities and roles, but by a unique quality of experience" ${ }^{4}$.

Adicionalmente, en esta etapa los niños y niñas empiezan a adquirir mayor conciencia de sus diferencias anatómicas y a incorporar estereotipos de género en sus conductas y juegos, haciendo uso de los códigos establecidos por género (Brill y Pepper, 2008). A esa edad, los roles de género se empiezan a refinar a partir de la interacción con otros y de lo que aprenden de sus familias, medios de comunicación y valores sociales. Siguiendo a estos mismos autores, los niños a esta edad, aunque vean a personas que actúan en roles que comúnmente se vinculan con el sexo opuesto, todavía tienden a dividir las conductas por género. Sin embargo, investigaciones sugieren que cuando a los niños de este tramo de edad se les entregan ejemplos distintos a través de cuentos o exposición a situaciones reales, ellos pueden adaptar sus constructos (Brill y Pepper, 2008; Gender Spectrum, 2014). Con suficiente información, niños de cualquier edad son capaces de comprender que hay más de dos categorías de género reconocidas por la sociedad, y cuando se les explica de una manera sencilla y apropiada a su edad, la diversidad de género es un concepto fácil de comprender (Gender Spectrum, 2014). De allí la importancia que tiene el sistema educativo en la promoción de la tolerancia hacia las diferencias de género desde la primera infancia.

En esta etapa, los padres o cuidadores probablemente se van a preocupar si su hijo prefiere jugar con muñecas y maquillaje que con camiones, o si una niña prefiere realizar actividades más rudas que estar jugando con barbies y usando un vestido rosado. Al igual que en los estadios anteriores, dado que es más probable que los niños transgéneros reciban presión para ajustarse a las normativas sociales, serían más propensos a sentir culpa de sus propios deseos y necesidades. Ellos todavía no comprenden que los problemas de género son malentendidos como problemas sociales (Ettner, 1999).

Posteriormente, de acuerdo a la teoría de Erikson, de los seis a los nueve años de edad los niños tienen que lidiar con nuevas demandas sociales y académicas, y pueden desarrollar un sentimiento de competencia si logran desarrollar ciertas habilidades $y$

\footnotetext{
${ }^{4}$ Niños y niñas se diferencian no solo por sus diferentes órganos, capacidades y roles, sino que también por la calidad de las experiencias propias (traducción propia)
} 
responder de manera efectiva a las tareas. Si un niño o niña recibe una retroalimentación positiva, él o ella se motivará y animará para continuar con nuevas tareas. Por el contrario, si reciben criticas constantemente, ellos pueden desarrollar un sentimiento de decepción e inferioridad, situación posible de experimentar por los niños transgéneros que de cierta forma no estarían cumpliendo con ciertas expectativas sociales.

Complementando las características de este estadio psicosocial con la literatura relativa a los niños transgéneros, cabe mencionar que en general a los siete años los niños que han mostrado una identidad de género cruzada por varios años, estarían dando a conocer que pueden ser transgéneros, aunque algunos niños deciden no desplegar su verdadera identidad de género hasta años posteriores (Kennedy \& Hellen, 2010).

Siguiendo a Erikson, el consiguiente impacto de una continua desaprobación y rechazo sobre la autoestima y los sentimientos de competencia de un niño en caso de no alcanzar una resolución adecuada de cada uno de los conflictos de las etapas mencionadas (Confianza básica Versus Desconfianza básica; Autonomía Versus Vergüenza y Duda; Iniciativa versus Culpa; Industria Versus Inferioridad) es comparable con aquellos efectos descritos por Brill y Pepper (2008) para aquellos niños cuya identidad de género ha sido reprimida y restringida por terceros, tales como un pobre sentido del sí mismo, depresión, ansiedad, miedo y rabia. Por ejemplo, en el caso de Mario, al ir creciendo empezó a exponerse a actividades de riesgo, bajar sus notas, y desarrollar actitudes desafiantes tanto en el colegio como en su casa. Esto coincide con las experiencias recopiladas en la escasa literatura en el tema, donde se señala que los niños transgéneros empiezan a desplegar conductas problemáticas para proteger el sí mismo, intentando en algunos casos desplazar sus sentimientos de inferioridad al convertirse en personas que quieren agradar o entretener constantemente a otros, o al desplegar conductas agresivas y hacer bullying a sus compañeros (Hopper, 2007), como Mario, quien al mismo tiempo que era víctima de rechazo, reaccionaba agresivamente contra terceros.

Mario también tenía serios problemas con los profesores, quienes juegan un papel crucial en la infancia, pero lejos de respetar su identidad de género lo exponían a situaciones sumamente vergonzosas, tales como preguntarle si usaba ropa de interior femenina o masculina. Dichos episodios humillantes no son extraños para los niños y niñas transgéneros (Gender Spectrum, 2014), situaciones ejemplificadas en la películas mencionadas en la primera parte de este ensayo, en historias de niños transgéneros descritas por medios de comunicación (Rosin, 2008) o en relatos de familiares (Mansilla, 2014). Otro problema surge cuando las conductas de riesgo son a menudo consideradas como evidencia de que algo malo sucede con el niño, como parte de la naturaleza de los niños transgéneros, en vez de comprender dichas conductas como una respuesta normal en el intento de acomodarse a un ambiente hostil (Mallon \& DeCrescenzo, 2006). De la misma manera, los profesionales fallan al desconocer la influencia de otros sistemas en dichas actitudes, tales como el colegio o el sistema de protección, culpando al niño o a la familia de estas acciones, (Oaklander, 2006). Finalmente, y es aún más preocupante, las descripciones negativas que se hacen en relación a las conductas disruptivas de los niños transgéneros o sobre sí mismos pasan a ser parte de la 
identidad del niño. Por ejemplo, Mario solía describirse a sí mismo como raro e inútil, lo que no es extraño, toda vez que sus profesores lo culpaban del desorden de la sala, y su padrastro de los conflictos maritales, al tiempo de referirse hacia él como un monstruo y la vergüenza de la familia, haciendo uso del castigo físico en caso de un comportamiento disruptivo. Sin embargo, el uso de la violencia para "curar" a Mario no alivió la crisis familiar, por el contrario, terminó agravándola.

\section{- Adolescencia: enfrentando los cambios corporales.}

Después de la niñez temprana y la pre pubertad, la adolescencia es la tercera época más común en que un niño o niña se da cuenta que es transgénero (Brill y Pepper, 2008), aunque no necesariamente se definan a sí mismos como tal, ya que en general hay un desconocimiento que pueden existir niños y niñas transgéneros. Esto se puede vincular al escaso número de especialistas y la falta de información que hace más difícil para los niños transgéneros y sus padres enfrentar estos cambios (Ettner, 1999). Este desconocimiento y falta de información queda en evidencia con los hallazgos de un estudio realizado con adultos transgéneros en el Reino Unido, que señalan que en promedio las personas no aprendieron las palabras que los describían hasta los 15,4 años. Una diferencia de 7,5 años desde que fueron conscientes de su identidad transgénero. Este descubrimiento fue significado por los participantes del estudio como un alivio (Kennedy y Hellen, 2010).

Siguiendo la teoría de los estadios del desarrollo psicosocial de Erikson, el desarrollo de la identidad personal caracteriza la etapa de la adolescencia, así como las luchas con las interacciones sociales y las cuestiones morales. Si un adolescente no es capaz de aceptarse a sí mismo, de encontrar un satisfactorio sentido del sí mismo, él o ella se verá confundido (Hopper, 2007), como Mario, quien entrando a la adolescencia empezó a golpear a su madre. Incluso la policía estaba interviniendo cuando los problemas se volvieron inmanejables.

Esta exacerbación de la confusión y la crisis familiar puede ser vinculada a la emergencia de los caracteres sexuales secundarios, un aspecto significativo de esta etapa. Mientras la mayoría de los niños tienden a aceptar dichos cambios superando ciertos inconvenientes, los niños transgéneros tienden a enfrentar dichos cambios con estrés, angustia, vergüenza y aversión (Brill y Pepper, 2008). Así como el caso de otros niños transgéneros, estos cambios gatillaron conductas autodestructivas en el caso de Mario. El solía esconder sus senos con desesperación por medio de una faja. A ello cabe añadir que la exploración sexual es también vivida por los niños transgéneros con mayor ansiedad, sobre todo cuando interactúan sexualmente con terceros que desconocen su sexo biológico.

\section{Las limitaciones de la teoría de Erikson para comprender la diversidad humana.}

Esta teoría, ampliamente abordada en el campo de la psicología del desarrollo, tiene varias limitaciones para comprender la realidad de los niños transgéneros. En primer lugar, no considera la existencia de conflictos de género en la infancia. En contraste a la teoría de Erikson, quien supone que los principales conflictos y crisis en torno a la 
identidad surgen en la adolescencia (Smith et al., 2003), las luchas que deben enfrentar los niños transgéneros nos llevan a entender que las crisis de identidad pueden aparecer en la primera infancia, cuando los niños y niñas aprenden acerca de su cuerpo, del sexo biológico y las expectativas y roles basados en el género (Grossman y D'Augelli, 2007), al tiempo que se dan cuenta que las percepciones que tienen de ellos mismos no coinciden con la percepción de los demás.

En segundo lugar, si bien Erikson tomó en cuenta la influencia del contexto en el desarrollo infantil, él explica las diferencias entre hombres y mujeres principalmente por aspectos biológicos en vez de aspectos sociales, construyendo su teoría en una concepción dualista del género y entendido éste como una extensión del cuerpo biológico: "one way in which sex permeates personality, as conceived by Erikson, is in his notion that a woman is never-not-a-woman, a man is never-not-a-man" ${ }^{5}$ (Franz y White, 1985, p. 227). Este concepto binario no logra dar cuenta de un abanico de posibilidades de ser hombre o mujer. En lugar del modelo estático y binario producido a través de una comprensión exclusivamente física del género, nuestra biología, nuestra expresión de género y nuestra identidad de género pueden cruzarse de maneras múltiples (Brill y Pepper, 2008). La diversidad de género es un término que reconoce que las preferencias de muchas personas y sus expresiones de género no pertenecen a lo que comúnmente hemos comprendido dentro de las normas de género, siendo parte de las expresiones humanas documentadas a lo largo de todas las culturas y la historia.

En tercer lugar, de acuerdo a Geldard y Geldard (2008), una integración estable del individuo de acuerdo a la teoría de Erikson está orientada a la identidad heterosexual, fallando en reconocer un amplio espectro de orientaciones sexuales.

En cuarto lugar, y siguiendo a Rose (1990), otra crítica posible a la teoría de Erikson, crítica que se puede hacer extensiva a la psicología del desarrollo, es que en general muestra un retrato de la normalidad para los niños a una edad. Esto permite a distintos profesionales evaluar la normalidad de un niño en comparación con esta norma (citado en Jenks, 2009), en detrimento de los niños que están fuera de esas normas construidas socialmente, como lo son las del género.

En quinto lugar, y como fue explicado anteriormente, para Erikson un sentido de competencia surge si un estadio es adecuadamente enfrentado, comparado a un sentimiento de incompetencia que surge si este es manejado pobremente (Hopper, 2007). Sin embargo, debido a la intolerancia en las sociedades frente a la diversidad de géneros, las trayectorias del desarrollo de los niños transgéneros para convertirse en adultos tienen más barreras y son más complicadas. Estas luchas nos llevan a suponer que el objetivo de una identidad estable no necesariamente se alcanza al final de la adolescencia, más aún si se asocia una identidad estable con una visión heteronormativa. Además, los adolescentes transgéneros se enfrentan a incertidumbres futuras que no les animan a alcanzar la adultez: ¿Es el comercio sexual el único lugar donde puedo trabajar? ¿Puede la persona que ame aceptarme cómo soy? Teniendo esto

\footnotetext{
${ }^{5}$ Una forma mediante la cual el sexo permea la personalidad, tal como lo concibe Erikson, es su noción de que una mujer no es nunca una no-mujer y un hombre nunca un no-hombre (traducción propia dela autora)
} 
en cuenta, se podría dar por sentado, y es la visión criticada en este ensayo, que los niños transgéneros son menos capaces de desarrollar una personalidad sana $\mathrm{e}$ interacciones sociales adecuadas, incluso cuando estos conflictos se pueden resolver con éxito más tarde.

Un enfoque teórico más amplio capaz de incorporar la diversidad de género parece ser entonces más pertinente. Esto es, un enfoque que comprenda el sentido de competencia como la integración de una identidad de género diversa en el sentido de sí mismo, un logro alcanzado por las personas transgéneros a menudo en la adultez más que en la adolescencia (Kennedy y Hellen, 2010). Por ejemplo, el enfoque psicoanalítico de Carlo Strenger $(1997,2000)$ quien defiende las múltiples construcciones de la individualidad, etapas de la vida, roles de género, clase de las familias y sexualidades (citado en Bohleber, 2010). A partir de dicho enfoque, otras alternativas de individualización para alcanzar la autorealización y la autenticidad son posibles (Bohleber, 2010), visión que puede ser considerada como negativa para algunos cientistas, es sin duda una oportunidad para la integración social de los niños transgéneros.

\section{El aporte del enfoque humanista para abordar la transgeneridad.}

Mario había visitado varios especialistas en materia de salud mental que trataron sus problemas de conducta, sin ayudarlo a comprender qué estaba pasando con él, incluso un profesional de la salud le ofreció realizar una prueba genética para confirmar si era hombre o mujer, lo que aumentó su angustia frente a la posibilidad que dicho examen corroborara que biológicamente era una mujer. Sin embargo, los comportamientos desplegados por Mario fueron definitivamente un grito no verbal de ayuda después de un largo tiempo de lucha con su identidad, sus relaciones sociales y su autoestima, que son algunas de las razones por las que los niños van a la terapia (Oaklander, 2006).

Si bien Mario no tuvo acceso a una terapia de calidad en su consultorio, debido a lo corto de ésta y a la rotación de profesionales, el tener acceso a un sistema de salud mental no es garantía suficiente. Los terapeutas deben estar preparados para hacer frente a esta realidad.

Aunque algunos elementos de la psicología del desarrollo son relevantes para ayudar a los niños transgéneros para consolidar el sí mismo a través de la resolución exitosa de las crisis de desarrollo (Geldard \& Geldard, 2008, p. 32), una perspectiva humanista centrada en ayudar a los niños a desarrollarse de acuerdo a su propia naturaleza, a aceptar sus diferencias y a conciliarse con el sí mismo, ha demostrado ser una perspectiva más adecuada que tratamientos de corte psicoanalítico (Ettner, 1999).

Aquí cabe destacar la perspectiva centrada en la persona de Carl Rogers fundada en la creencia de que los seres humanos tienen una tendencia inherente hacia el crecimiento, el desarrollo y el funcionamiento óptimo (Nash, 2008). A través de una relación terapéutica que no juzga y es cálida, es posible sugerir que los niños transgéneros pueden alcanzar una verdadera aceptación del sí mismo y desarrollar su potencial. Acá 
el papel del terapeuta es facilitar un espacio seguro donde esta tendencia se puede trabajar de forma constructiva (Bryant-Jefferies, 2004) y evitar que sea distorsionada por las condiciones desfavorables (McMillan, 2004).

\section{El apoyo a los niños y niñas transgéneros en el contexto escolar.}

Es posible señalar que hay un alta probabilidad que los niños transgéneros sean derivados a espacios terapéuticos o consejerías dentro de sus establecimientos escolares (en los que los hay) ya que ellos son más susceptibles de ser juzgados y de ser víctimas de bullying (Brill y Pepper, 2008). Ser transgénero en una sociedad que discrimina la diversidad de géneros puede gatillar sentimientos de vergüenza, aislamiento, desconfianza y un sentido negativo del sí mismo que requieren un apoyo adicional.

Vale la pena destacar un debate en relación al objetivo del tratamiento que puede recibir un niño o niña transgénero. Persuadir a los niños para ocultar sus propios deseos, a fin de cumplir con las expectativas sociales, puede llegar a ser una alternativa sumamente destructiva que puede aumentar la confusión de los niños. Del mismo modo, esta práctica no es considerada ética en países anglosajones (BACP, 2010; COSCA, 2011;WPATH, 2012), y ha demostrado su ineficacia, a diferencia de las estrategias encaminadas a apoyar a los niños transgéneros a desarrollar la confianza en sí mismos y su integración social, ofreciendo una plataforma de reflexión sobre las múltiples opciones relacionadas con la expresión del género y la identidad (Malpas, 2011). Asimismo, se considera relevante apoyar a los niños a comprender que el ser transgénero es parte de la diversidad humana, normalizando sus experiencias. En este segundo enfoque, el cambio viene por medio de la liberación de lo que ya existe en el sujeto (McMillan, 2004). Para ello es recomendable que los terapeutas manifiesten una disponibilidad a comprender y aceptar las distintas experiencias de los seres humanos (Bohleber, 2010) y sean capaces de reconocer los costos sociales, culturales y políticos de ser transgénero para evitar una patologización de los síntomas. Se trata de apoyar su empoderamiento (Lev, 2000), la realización de su máximo potencial y la búsqueda de encontrar la comodidad con su identidad de género y su cuerpo (Ettner, 1999), para fortalecer y mejorar sus relaciones sociales. Los terapeutas deben examinar sus propios prejuicios para evitar una doble victimización y un contratransferencia negativa (Hopper, 2007), junto con ser sensibles, empáticos y respetuosos frente a las múltiples configuraciones del sí mismo.

Los terapeutas también deben tener en cuenta que los niños transgéneros no son un grupo homogéneo, y ser transgénero masculino o femenino tiene diferentes implicaciones sociales y culturales. Además, una condición previa es respetar las preferencias de los niños transgéneros asociadas a su propia identidad de género, tales como sus elecciones en cuanto a ropa, corte de pelo y nombre (Brill y Pepper, 2008). Por otra parte, los terapeutas tienen que respetar los tiempos de cada proceso, ya que empujar a un niño a aceptar una identidad transgénero y asumir una transición podría ser aún más perjudicial. Una transición se refiere al período que el niño revela, se apropia y asume una identidad de género cruzada frente a otros, debiendo estar preparado para todo tipo de reacciones (Brill y Pepper, 2008; Lev, 2000). En este 
contexto los terapeutas tienen que desarrollar las competencias para más tarde orientarlos si deciden someterse a tratamientos de hormonas o una cirugía de reasignación de sexo, con el fin de hacer frente a estos cambios (Malpas, 2011).

Barwick (2002) describe cómo las terapias en las escuelas a menudo pueden ser un refugio en la última parada antes de la exclusión (citado en Hawkins, 2008) y convertirse en un espacio donde los niños pueden confiar sus aflicciones, encontrar significado a sus experiencias (Lev, 2000), aliviar la culpa de la tensión que sus expresiones de género han provocado en su familia (Brill y Pepper 2008), fortalecer su autoestima y desarrollar sus capacidades resilientes para hacer frente a la discriminación.

Junto a ello, algunos autores (Chen-Hayes, 2001; Lev, 2000; Malpas, 2011) recomiendan un rol más amplio de los terapeutas que trabajan con los niños transgéneros en los colegios, debido a la falta de capacitación de padres y profesores para atender los temas vinculados a la diversidad de género. A pesar de que el canon más apreciado en psicoterapia es que el terapeuta permita al cliente encontrar sus propias soluciones frente a los problemas, el trabajo con los niños transgéneros a menudo requiere violar este principio (Ettner, 1999). Brill y Pepper (2008) sugieren que los terapeutas deben involucrar a profesores y padres con el fin de ampliar una red de aceptación, con tal de impedir nuevos abusos y conductas de riesgo, y al mismo tiempo que se respete la privacidad y confidencialidad de la terapia. Cualquier revelación debe incorporar el permiso del niño o niña. Además, el terapeuta debe ser consciente de que algunas dificultades pueden aparecer en la interacción con otros profesionales (Mabey y Sorensen, 1995), como las presiones para obligar al niño o niña a cumplir las expectativas de género.

Dada la influencia de los profesores sobre los niños transgéneros y sus compañeros, es recomendable proporcionarles herramientas para atender temas relativos a la diversidad de las expresiones de género en el aula (Brill y Pepper 2008), defendiendo una política de tolerancia cero a la discriminación a través de la promoción de una cultura organizacional de apoyo escolar. Teniendo en cuenta que las escuelas suelen tener reglas rígidas por género, es importante promover una mayor flexibilidad para garantizar que todas las identidades de género sean tratados con dignidad (Chen-Hayes, 2001).

De la misma manera, dado que los niños transgéneros tienden a "perturbar" a quienes normalizan la polaridad de las expresiones de género, especialmente a los padres (Mallon y DeCrescenzo, 2006), es importante que los terapeutas puedan guiar a los padres a una verdadera aceptación de sus hijos, quienes sin excepción necesitan sentir que sus padres creen y confían en ellos. Además, algunos padres podrían necesitar ayuda para manejar las conductas del niño a través estrategias que no avergüencen a sus hijos y terminen erosionando su autoestima (Ettner, 1999). Del mismo modo, los terapeutas deben ser sensibles al impacto en la familia de tener un integrante transgénero y el impacto que pueden generar la transición del niño (Lev, 2000). En vista de los temores de los padres respecto al bienestar futuro de sus hijos, el miedo a la condena, la pena, la auto-culpa y sentimientos de pérdida y vergüenza (Brill y Pepper, 2008), pueden necesitar ser derivados a otro apoyo psicológico. 
Sin lugar a dudas, atender a niños que han tenido que traicionar al sí mismo para complacer a los demás, ser objeto de rechazo y vivir con la vergüenza (Ettner, 1999) es una tarea difícil. Aún más teniendo en cuenta que la vergüenza interfiere con otras actividades y en la formación del carácter. Por lo tanto ayudar a los niños a comprender la incongruencia de su ser interno y externo (Ettner, 1999) y pasar de una experiencia del rechazo al respeto del sí mismo (Lev, 2000) puede llegar a ser una experiencia de liberación transcendental de los niños transgéneros. Ellos y ellas no nacieron en el cuerpo equivocado. Una mayor apertura a la diversidad nos permite comprender que no hay una correlación exacta entre nuestro sexo y el género, lo que es vital de trasmitir a los niños transgéneros. Su cuerpo es igual de valioso que los otros cuerpos, es la sociedad la que se ha equivocado en discriminarlos.

\section{La importancia de las intervenciones tempranas.}

Después del diagnóstico y debido a la falta de recursos económicos, Mario volvió a ser derivado al sistema público de salud mental. Al año siguiente, Mario falleció al estar practicando una actividad ilegal de alto riesgo. Es entonces cuando surgen las siguientes preguntas ¿si Mario hubiera recibido el apoyo que necesitaba de manera oportuna, se hubiera involucrado en actividades de alto riesgo? ¿Si el sistema de protección le hubiera brindado el apoyo que requería él y su familia, podría haber vivido?

\section{Conclusiones y reflexiones finales}

Los roles y expectativas sociales de género están tan arraigados en nuestra cultura que la mayoría de la gente no puede imaginar ninguna otra manera de concebir el género. Como resultado, la mayor parte de los individuos encajan en estas expectativas y rara vez cuestionan qué significa realmente el género, porque el sistema generalmente ha funcionado para ellos, sin embargo para algunas personas es distinto

Si bien la mayoría de las personas desarrollan una identidad de género que coincide con su sexo biológico, para algunos niños y niñas su identidad de género es diferente a su sexo. Algunos niños pueden tener un sentimiento intuitivo que su identidad de género no coincide con el género asignado al nacer. Como padres, verse en esta situación puede ser uno de los mayores desafíos a enfrentar. Nadie espera que su hijo o hija no actúe de acuerdo al género asignado al nacer. Sin embargo, en la realidad algunos niños no están conformes con dicha asignación, y esa disconformidad de género puede mantenerse en el tiempo. Si bien para algunos esa disconformidad puede ser una fase, para otros niños no lo es. La respuesta se va a clarificar en el tiempo. Independientemente del resultado final, la autoestima, el bienestar y la salud en general de un niño o niña que no está conforme con el género asignado al nacer se basará en gran medida en el amor, apoyo y aceptación incondicional de sus padres.

Las personas transgéneros han demostrado que núcleos centrales del sí mismo, como el sexo biológico y la identidad de género, son diferentes. Sin embargo, al romper la construcción binaria y hegemónica del género, ellos y ellas se han convertido en parte de los grupos más excluidos, muchos de ellos prácticamente limitados a desarrollarse 
laboralmente sólo en el comercio sexual.

Ahora, si ser transgénero es particularmente difícil, ser un niño transgénero lo es aún más dada la existencia de un gran desconocimiento de esta realidad. Sin embargo, se ha prestado poca atención a cómo las familias, los profesores y terapeutas pueden apoyar las distintas expresiones de género. Una tendencia a la patologización no ayuda a cambiar esta situación.

Es importante comprender que la diversidad de género no es una enfermedad o un resultado de la mala crianza. No es el resultado de un divorcio o un indicador de abuso sexual. La diversidad de género no es causada por la crianza liberal, o permisiva, o por un padre que secretamente deseaba que su hijo fuera el sexo "opuesto". Es normal. La investigación actual apoya que hay explicaciones biológicas que permitirían comprender por qué esto sucede (Brill y Papper, 2008). Por lo tanto, los padres no pueden provocar que su hijo no actúe conforme a su sexo ni tampoco puede intervenir para que un niño cambie su identidad de género y actúe conforme a su sexo; pero, y es vital, sí pueden tener un profundo impacto en el bienestar de sus hijos y en cuanto a cómo ellos se sienten en relación a sí mismos en la medida que los acepten tal y como ellos se expresan.

Las teorías del desarrollo que se sustentan en una heteronormatividad pueden profundizar la estigmatización sufrida por los niños transgéneros, quienes tienden a desplegar varios síntomas y comportamientos que deben ser entendidos como estrategias para afrontar el dolor de ser objeto de discriminación, en lugar de una parte de su naturaleza transgénero. Por el contrario, el enfoque humanista centrado en la persona ofrece una oportunidad para que los niños transgéneros puedan transformar la percepción acerca del sí mismo a través de una relación terapéutica basada en la aceptación y la confianza (Hawkins, 2008). Un auténtico reconocimiento de la amplia gama de expresiones humanas y la singularidad de los niños son elementos primordiales. Por el contrario, cualquier intento de obligar a los niños a suprimir su identidad de género con el fin de cumplir con las expectativas sociales más aceptadas ha demostrado ser infructuoso y poco ético.

La propuesta de trabajo con niños transgéneros acá planteada propone un rol más amplio del terapeuta frente al posible estado de confusión en que los padres podrían estar inmersos, a la probabilidad que los niños transgéneros puedan involucrarse en conductas de riesgo para ellos o para terceros y de ser víctimas de bullying en los colegios. Independientemente de las capacidades de los padres y los profesores, la mayoría de ellos no están preparados para hacer frente a la diversidad de género y necesitan orientación para avanzar hacia una mayor integración y aceptación de los niños transgéneros en los colegios y en sus familias. Las políticas públicas orientadas a fomentar la equidad de género dentro de los establecimientos escolares tienen bastante que decir al respecto.

Por último, se requiere más investigación para apoyar la formación de profesionales de distinta índole en el trabajo con niños transgéneros, siendo importante difundir estos conocimientos a los países donde las prácticas y estructuras discriminatorias hacen 
considerablemente más difícil la integración de las personas transgéneros. Del mismo modo, los terapeutas deben abogar por el reconocimiento social de los niños transgéneros, que han sido inmensamente valientes por defender su verdadero ser en un ambiente hostil. El reconocimiento podría haber cambiado la trayectoria de vida que tuvo Mario.

\section{Bibliografía}

BACP (British Association for Counselling and Psychoteraphy) (2010) Ethical Framework for Good Practice in Counselling and Psychotherapy. [On Line]. Disponible en: http://www.bacp.co.uk/ethical_framework/. Accedido el 13 de enero del 2013.

Bohleber, W (2010) Destructiveness, intersubjectivity, and trauma: the identity crisis of modern psychoanalysis. Londres: Karnac.

Bryant-Jefferies, R (2004) Counselling Young People. Person-Centred Dialogues. Abingdon: Radcliffe Medical Press.

Brill, S y Pepper, R (2008) The Transgender Child: A Handbook for Families and Professionals. San Francisco: Cleis Press Inc.

Chen-Hayes, S (2001) 'Counseling and Advocacy With Transgendered and GenderVariant Persons in Schools and Families', Journal of Humanistic Counseling, Education and Development 40 (1), 34-49.

Conroy, M (2010) 'Treating Transgendered children: Clinical Methods and religious mythology', Zygon, Journal of Religion and Science 45 (2), 301-316.

COSCA (Counselling and Psychoteraphy in Scotland) (2011) Statement of Ethics and Code of Practice. [On Line]. Disponible en http://www.cosca.org.uk/new documents.php?headingno=2\&heading=Ethics Accedido el 13 de enero del 2013

APA (American Psychiatric Association's) (2013) Diagnostic and statistical manual of mental disorders-DSM-V. [versión electrónica] : Washington, D.C. : American Psychiatric Publishing.

Ettner, R (1999) Gender Loving Care. A guide to Counseling Gender- Variant Clients. Nueva York: W.W. Norton and Co.

Franz, C y White, K. (1985) 'Individuation and attachment in personality development: Extending Erikson's theory'. Journal of Personality , 53 (2), 224-256.

Geldard, K y Geldard, D (2008) Counselling Children: A Practical Introduction. Londres: Sage.

Gender Spectrum (2014) Resources. [On Line]. Disponible en: https://www.genderspectrum.org/resources/ Accedido el 27 de mayo de 2015.

Grossman, A y D'Augelli, A (2007) 'Transgender Youth and Life-Threatening Behaviors', Suicide and Life-Threatening Behavior 37 (5), 527-537. 
Hawkins, S (2008) Working at relational depth with adolescents in schools: a personcentred psychologist's perspective. En S. Keys y T. Walshaw (eds), Personcentred work with children and young people: UK practitioner perspectives (pp. 47-57). Ross-on- Wye: PCCS Books.

Hopper, L (2007) Counselling and psychoterapy with children and adolescents. Houndmills, UK: Palgrave Macmillan.

Jenks, C (2009) Constructing childhood sociologically. En M. J. Kehily, y M. G. Press (Eds.), An introduction to Childhood Studies (2 ed., pp. 93-112). Maidenhead,UK: Open University Press.

Kennedy, $N$ y Hellen, $M$ (2010) 'Transgender children: more than a theoretical challenge', Graduate Journal of Social Science 7 (2), 25-43.

Lev, A (2000) Transgender Emergence: Understanding Diverse Gender Identities and Expressions. [On Line]. Disponible en: http://www.choicesconsulting.com/assets/pro writing/transgender\%5B1\%5D.p df Accedido el 15 de febrero del 2013.

Lev, A (2005) 'Disordering Gender Identity: Gender Identity Disorder in the DSM-IV-TR', Journal of Psychology and Human Sexuality 17 (3/4), 35-69.

Mabey, J y Sorensen, B (1995) Counselling for young people. Buckingham: Open University Press.

Mallon, G y DeCrescenzo, T (2006) 'Transgender Children and Youth: A Child Welfare Practice Perspective', Child Welfare 85 (2), 215-241.

Malpas, J (2011) 'Between Pink and Blue: A Multi-Dimensional Family Approach to Gender Nonconforming Children and their Families', Family Process 50 (4), 453470.

Mansilla, G (2014) Yo Nena, Yo Princesa. Luana, la niña que eligió su propio nombre. Los Polvorines: Ediciones UNGS.

Martínez-Guzmán, A y Montenegro, M ( 2010) 'Narrativas en torno al Trastorno de Identidad Sexual. De la multiplicidad transgénero a la producción de transconocimientos', Prisma Social (4), 1-44. . [On Line]. Disponible en:

http://www.isdfundacion.org/publicaciones/revista/pdf/03 N4 PrismaSocial antar ma risela.pdfAccedido el 27 de septiembre de 2015.

McMillan, M (2004) The person-centred approach to therapeutic change. Londres: Sage.

MOVILH (Movimiento de Liberación Homosexual) (2010) 'Educando en la Diversidad Orientación sexual e identidad de género en las aulas'. [On Line]. Disponible en:

http://www.movilh.cl/documentacion/educando en la diversidad 2da edicion web.p dfAccedido el 27 de mayo de 2015. 
Nash, S (2008) Exploring issues of bereavement and loss with children and young people. A person-centred perspective. En S. Keys y T. Walshaw (eds), PersonCentred work with Children and Young People. UK practitioner perspectives (pp. 123-136). Ross-on- Wye: PCCS Books.

Oaklander, V (2006) Hidden treasure a map to the child's inner self. Londres: Karnac.

Smith, P, Cowie, H, y Blades, M (2003) Understanding Children Development $\left(4^{\text {th }}\right.$ ed.). Oxford: Blackwell Pub.

Rosin, H (2008) 'A boy's life'.Atlantic Monthly (10727825) 302 (4), 56-71.

Wester, S, McDonough, T, Maureen, W, Vogel, D y Taylor, L (2010) 'Using Gender Role Conflict Theory in Counseling Male-to-Female Transgender Individuals'. Journal of Counseling and Development , 88 (2), 214-219.

WPATH (The World Professional Association for Transgender Health) (2012). Standards of Care for the Health of Transsexual, Transgender, and Gender Nonconforming People ( $7^{\text {th }}$ version). [On Line]. Disponible en: http://www.wpath.org/: http://www.wpath.org/documents/SOC\%20V7\%2003-17-12.pdf. Accedido el 10 de febrero del 2013

Fecha de recepción: 30 de julio de 2014

Fecha de aprobación: 7 de octubre de 2015 